\title{
Case report: Metastatic adenocarcinoma of the lung with filiform "ciliated" tumor cells in the cerebrospinal fluid
}

\author{
Daniel A. Zlotoff ${ }^{1}$, Cynthia Kelly², Malca Kierson ${ }^{2}$, Cindy McGrath ${ }^{2}$, Prabodh Gupta ${ }^{2^{*}}$ \\ ${ }^{1}$ Department of Medicine, Perelman School of Medicine at the University of Pennsylvania, Philadelphia, USA \\ ${ }^{2}$ Department of Pathology and Laboratory Medicine, Perelman School of Medicine at the University of Pennsylvania, Philadelphia, \\ USA; *Corresponding Author: Prabodh.Gupta@uphs.upenn.edu
}

Received 13 January 2013; revised 2 March 2013; accepted 4 April 2013

Copyright (c) 2013 Daniel A. Zlotoff et al. This is an open access article distributed under the Creative Commons Attribution License, which permits unrestricted use, distribution, and reproduction in any medium, provided the original work is properly cited.

\begin{abstract}
Occurrence of cilia on the cell surface is usually considered evidence of a non-neoplastic process. Rarely, ciliated malignant tumors cells have been reported in pleural and ascitic fluids from ovarian and endometrial adenocarcinomas, though not from the cerebrospinal fluid (CSF) in association with pulmonary adenocarcinoma. We now present a case report of a patient who initially presented with neurologic symptoms. A CSF specimen revealed atypical cells with peripheral cilia and some cytoplasmic pigment. Cytologic, morphologic, and immunohistochemical analyses established this to be a metastatic adenocarcinoma of pulmonary origin with metastases to the meninges, pelvis, and the vertebrae. Cilia are generally the antithesis of malignancy as their presence is considered to be evidence of proper regulation of the cell cycle within a well-differentiated cell. This case offers the first described example of malignant ciliated cells derived from a metastatic lung adenocarcinoma. An awareness of this unusual presentation should aid in diagnosis and management when similar situations are prospectively encountered.
\end{abstract}

Keywords: Adenocarcinoma; Lung; Cilia; CSF

\section{INTRODUCTION}

Cytologic analysis is critical for discriminating between benign and malignant cells. Various features can be used in this process, including nuclear:cytoplasmic $(\mathrm{N}: \mathrm{C})$ ratios, nuclear morphology, and the presence or absence of structures normally found on benign cells. Cilia are microtubule-based cellular structures used to generate extracellular fluid movement; in humans, they are primarily found on respiratory and fallopian tube epithelial cells. The presence of cilia is almost always associated with benign cells as their presence is considered a reflection of complete differential and normal cell cycle control. Case reports have identified cilia on malignant cells [1-7]. These reports have described ciliated cells in association with both gynecologic (endometrial and ovarian) and pulmonary primary tumors. To our knowledge, there are no published reports of ciliated metastatic lung adenocarcinoma.

We now describe a case of a 52 years old woman initially presenting with mental status changes whose CSF revealed ciliated malignant cells. Additional workup revealed a left upper lobe lung mass with sclerotic lesions of the pelvis and the L4 vertebra concerning for metastases. Brochioalveolar lavage yielded ciliated cells that were morphologically similar to those recovered from the CSF. Immunohistochemical analysis revealed the tumor cells to be positive for AE1/AE3, TTF-1 (nuclear), CK5/6 (weak focal positive staining), CD68, S-100, and napsin; cells were negative for p63. A diagnosis of primary lung adenocarcinoma with distant metastases of the meninges, pelvis, and L4 vertebra was made. This report thus stands as the first description of ciliated metastatic lung adenocarcinoma.

\section{CASE REPORT}

A 52 years old African-American woman presented to the Emergency Department of our hospital with acute mental status changes, expressive and receptive aphasia, and combativeness. Her past medical history was remarkable for asthma, depression and cocaine abuse; she 
was HIV negative. The patient was intubated and transferred to the medical intensive care unit (MICU) for further evaluation. A magnetic resonance image (MRI) of her brain demonstrated diffuse leptomeningeal enhancement with nodular extra-axial enhancement at the lateral aspect of the left supraorbital gyrus and other findings consistent with infectious meningioencephalitis. The clinical impression was possible herpes simplex virus (HSV) encephalitis and she underwent a lumbar puncture. The cerebrospinal fluid (CSF) results were compatible with aseptic meningitis and she began treatment with acyclovir. Cultures from this fluid were eventually negative for HSV, VZV, and Cryptococcus. Fungal and bacterial stains were negative.

A CSF specimen submitted for cytology was processed on a cellulose membrane (Millipore (C) filter) and cytospin preparations. Intermixed with a few lymphocytes and macrophages were numerous large (12 - 15 m) atypical cells occurring singly and in small clusters (Figures 1(A) and (B)), These atypical cells had normal to high nuclear: cytoplasmic (N:C) ratio (Figures 1(B)-(D), and $(\mathbf{F})$ ). The nuclei had notched contours with stippled to vesicular chromatin pattern and mild to moderate atypia (Figures 1(A)-(D)). A number of cells possessed ciliated structures of uniform length and periodicity, appearing as a brush border (Figures 1(A)-(C)); a terminal bar could be recognized in an occasional cell (Figure 1(B)). These ciliary tufts occurred along the cell border or at one end (Figures 1(A) and (B)). At places intracytoplasmic extension of the ciliary structures could be observed (Figure 1(C)); they were preserved in the paraffin-processed cells as well as immunohistochemical stained cells (Figures 1(E), (I), and (J)). While the biphasic cytoplasm with fine granularity was present in many cells, golden brown pigment granules were concentrated in the perinuclear hoff areas as well as throughout the cytoplasm of some cells, possibly representing intracytoplasmic pigment (Figure 1(D)).

Based upon these morphologic features, diagnostic consideration was given to cellular degeneration, specimen processing artifact, choroid plexus cells, and meningoendothelial cells, as well as malignancies such as an atypical histiocytic proliferation (histiocytosis), meningeal melanosis, and metastatic carcinoma. It was felt that these filiform "ciliated" structures did not represent the common artifact of blebbing of cytoplasmic borders since they were seen on Millipore $\subset$ filter preparations which result in minimal cellular deformation, artifacts, and damage. Additional diagnostic work-up to rule out a metastatic disease process was performed.

A chest X-ray revealed a left upper and lower lobe infiltrate and was interpreted as pneumonia. A contrastenhanced chest CT scan was performed subsequently which demonstrated atelectasis of the left upper lobe with adjacent pneumonia and ill-defined ground glass opacities and mediastinal lymphadenopathy. Based on the radiographic findings, the clinical impression was pneumonia, though malignancy and lymphoma could not be entirely excluded and a bronchoscopic examination was advised. A CT scan of the pelvis revealed multiple areas of sclerotic bone within the left iliac bone $(3.2 \times$ $1.9 \mathrm{~cm})$, right sacral ala $(1.9 \times 1.4 \mathrm{~cm})$, and the $\mathrm{L} 4$ vertebral body $(3.6 \times 2.6 \mathrm{~cm})$ that were suspicious for metastasis. No other potential sites of primary malignancy were identified within the abdomen or pelvis.

An adenocarcinoma with acinar and solid pattern was diagnosed in a bronchial wash specimen (Figure 1(F)). Bronchioalveolar lavage (BAL), a transbronchial biopsy of the left upper lobe, and a bone biopsy of the L4 vertebral body were performed. The material obtained from the transbronchial biopsy contained atypical epithelial cells demonstrating an acinar and solid pattern. The tumor cells from the L4 vertebral bone biopsy were morphologically similar to cells in the prior specimens and also possessed the same immunoprofile as the transbronchial biopsy specimen. All specimens contained tumor cells morphologically similar to the ones reported in the initial CSF specimen which exhibited more pronounced nuclear atypia and the presence of unipolar ciliated structures. Based upon the morphology and the in-situ component, a primary lung cancer was favored. Tumor cells stained positive for AE1/AE3 and TTF-1 (nuclear) and were weakly focally positive for CK5/6; cells were negative for p63 using immunohistochemical (IHC) stains. A diagnosis of a poorly differentiated adenocarcinoma with acinar features most compatible with lung primary was rendered.

As part of the clinical management of the patient's newly diagnosed metastatic lung adenocarcinoma, whole brain irradiation was considered. Although the CSF cells were morphologically similar to those obtained from the lung, a repeat lumbar puncture was performed to confirm that these cells were indeed metastases from the primary lung adenocarcinoma. Tumor cells in the CSF specimen revealed nuclear TTF-1 (Figure 1(G)) as well as AE1/ AE3 cytoplasmic staining. Bronchial washing material was also stained for macrophage marker (CD68), and was positive in tumor cells (Figure 1(H)). Bronchial wash cells demonstrated peripheral localization of cilia as seen by EMA immunohistochemical stain (Figures 1(I)-(K)). The tumor cells stained positive for napsin (Figure 1(L)), as well as S-100. Molecular studies revealed an EGFR mutation (exon 19 deletion). Electron microscopy could not be performed due to limited specimen. The patient has been treated for non-small cell carcinoma, including brain irradiation. She has responded well to the treatment and a chest CT scan performed after 3 months shows significant improvement in the left upper lobe mass. 


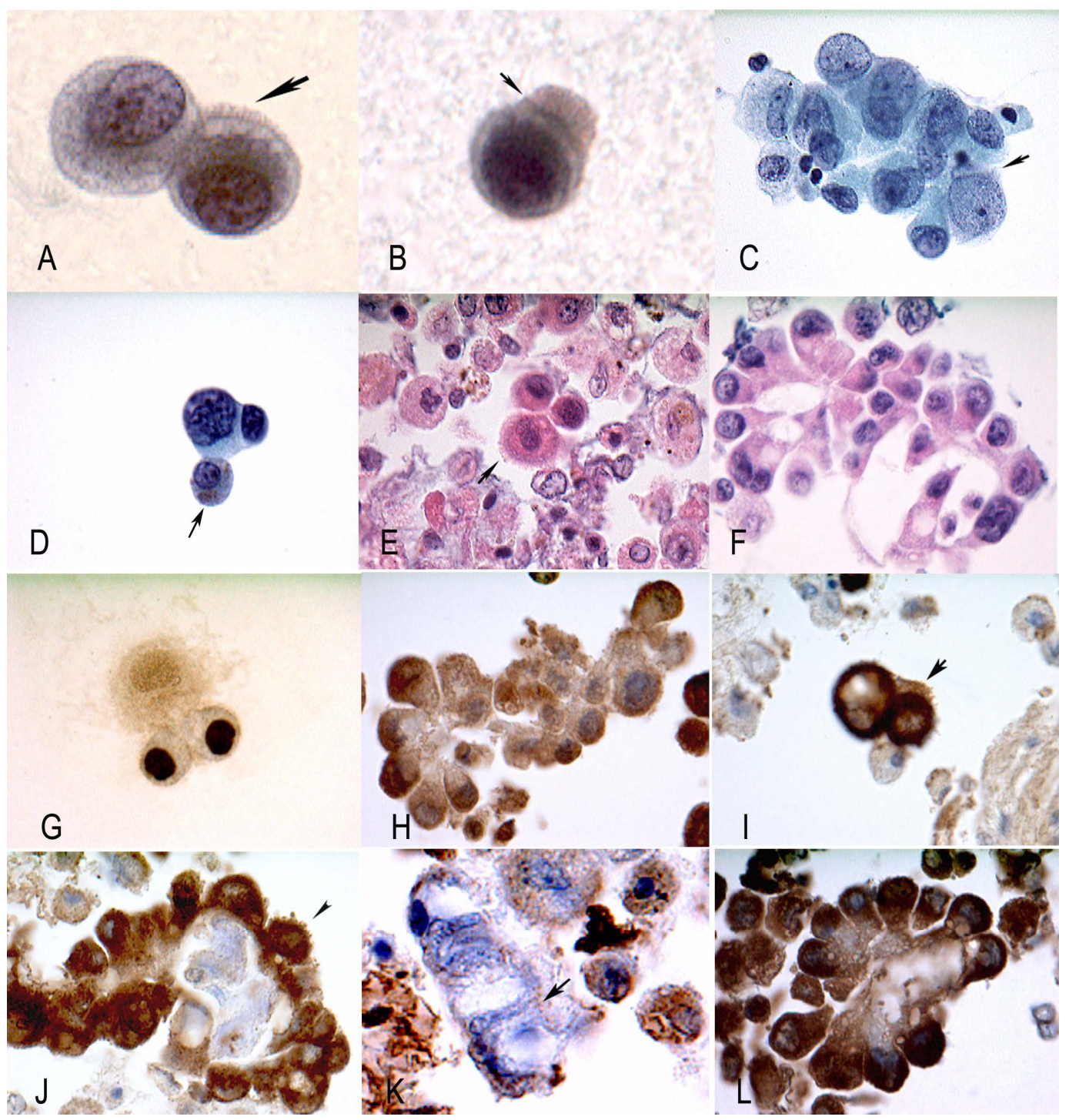

Figure 1. (A). Tumor cells, notice the ciliary structures (arrow). They are of uniform length, thickness, and periodicity. The cytoplasm is biphasic with peripheral rarefaction and granularity. Nuclei reveal hyperchromasia and chromatin changes. (B) Tumor cell; notice the ciliary tuft on one end and what appears to be the terminal plate (arrow), associated with true cilia. Cerebrospinal fluid (CSF) specimen, (A) and (B) Millipore filter, Papanicolaou stain HP. (C) Tumor cells with intracytoplasmic ciliary structures (arrow), CSF specimen, cyto- spin preparation, Pap stain. (D) Tumor cell; notice the intracytoplasmic, granular, brownish pigment (arrow) and nuclear features of malignancy. Such observations raise the possibility of melanoma. Cytospin preparation. Papanicolaou stain, HP. (E) Cytoplasmic cilia (arrow) cell block H/E, bronchial wash specimen, MP. (F) Adenocarcinoma. Bronchial wash, cells block H/E, MP. (G) Intranuclear localization TTF-1 immunostain, CSF specimen, Cytospin preparation, HP. (H) Cytoplasmic staining CD68, immunostain, bronchial wash, cell block, MP. (I) Peripheral localization, cilia (arrow), bronchial wash, cell block EMA immunostain, MP. (J) Peripheral localization cilia (arrow), adenocarcinoma lung, bronchial wash, cell block. EMA immunostain, MP. (K) Ciliated columnar cells, intracytoplasmic localization of ciliary roots, notice the dots (arrow), bron- chial wash. EMA immunostain, HP. (L) Cytoplasmic stain, papillary adenocarcinoma, bronchial wash, cell block, Napsin immunostain, MP.

\section{DISCUSSION}

Presence of true cilia among epithelial cells is considered an evidence of a benign non-neoplastic process. Malignant cells with ciliated structures are rare with only a few case reports in the literature. We present the first case in the English literature of a metastatic pulmonary adenocarcinoma with malignant cells with filiform ciliated structures identified by light microscopy in CSF, bronchial, and bone specimens. The presence of malignant cells with ciliated structures has been demonstrated 
by ultra-structural evaluation with electron microscopy and, more rarely, by light microscopy [5,7]. Tumor cells with ciliated structures are a diagnostic challenge in cytologic specimens as their presence is equated to benignity and the morphologic features of the cells are not always overtly malignant. Cilia are generally the antithesis of malignancy as their presence is considered evidence of proper regulation of the cell cycle within a well-differentiated cell, but as Koss and Melamed have aptly noted there are exceptions to this rule and ciliated cells can occasionally be malignant [1].

The normal distribution of ciliated epithelium is predominantly within the gynecologic and the respiratory tracts. Although easily recognizable, cilia exist frequently within benign tumors and metaplastic epithelium; when it is present in carcinoma, it is usually associated with well-differentiated, low grade tumors. Eichhorn and Scully described endometrioid ciliated cell tumors of the ovary [2]. Another case series by Hendrickson and Kempson [3] also highlighted criteria for distinguishing between the ciliated carcinoma variant of endometrial adenocarcinoma and benign ciliated lesions of the endometrium. Each of these reports identified cilia in histologic sections by light microscopy while the Hendrickson report also performed electron microscopy to confirm the presence of cilia. The first case report of ciliated malignant cells observed in a cytologic specimen was by Gupta et al. [5]. They described ciliated ovarian adenocarcinoma cells with malignant features occurring in ascetic and pleural fluid. The final resected ovarian specimen demonstrated poorly preserved cilia in several malignant cells in tissue sections.

The ciliated muconodular papillary tumor of the lung is a newly described entity by Sato et al. [2]. In this report of two cases, the surgically resected tumors on light microscopy demonstrate mild nuclear atypia with ciliated cells and no mitotic activity. Nakamura et al. [6] described an extremely well-differentiated papillary adenocarcinoma with prominent cilia formation in a $1.1 \mathrm{~cm}$ unencapusulated tumor. The tumor had papillary formations composed of cells with bland nuclear features and cilia. A report by Imai et al. [7] discusses the difficulty of preoperative cytology of a later resected peripheral pulmonary papillary adenocarcinoma with prominent cilia. Although the cells demonstrated moderate nuclear atypia they were interpreted as reactive. Retrospective comparison of the cells obtained on cytology and the surgically resected adenocarcinoma indicated that the cells were morphologically similar. Both of these case reports confirmed the presence of cilia by electron microscopy.

Occurrence of CD68 positivity within the malignant epithelial tumor cells is well documented [8]. Macrophages and cells rich in lysosomes/endosomes are stain- able with CD68. S-100 positivity in lung adenocarcinoma is well documented [9]. EMA is considered helpful in localization of terminal insertion of cilia (Figure

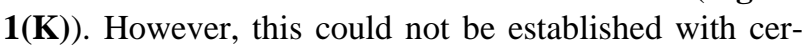
tainty in the present case. Although malignant cells can acquire phagocytic properties and can ingest foreign material, the presence of intracytoplasmic pigment within the malignant cells in the CSF specimen cannot be easily explained.

Occurrence of cilia in malignant cells is highly unusual, as this finding more often equates to a benign process. We present a rare case of metastatic lung adenocarcinoma with ciliated structures seen on light microscopy in both cytologic and histologic specimens. It remains to be determined if this is the first case of a ciliated lung adenocarcinoma with documented metastatic disease, as the primary tumor of the left upper lobe has not been resected. In case reports regarding this entity, the adenocarcinoma had an indolent course. The one exception, described by Imai et al. [7], was a patient whose tumor could be only partially excised due to the diffuse pleural spread of the tumor. Unlike the case presented here, none of these cases had evidence of distant metastases.

\section{CONCLUSION}

This case report describes a rare case of metastatic lung adenocarcinoma with ciliated malignant cells found in CSF, bronchial wash, and bone biopsy specimens. Though most often reflective of benign cells, the presence of cilia should not discount the possibility of malignancy. Healthcare providers should be aware that features of differentiated cells may be found in the context of malignant neoplasms.

\section{REFERENCES}

[1] Koss, L.G. and Melamed, M.R. (2005) Koss’ diagnostic cytology and its histopathologic bases. 5th Edition, JB Lippincott, Philadelphia.

[2] Sato, S., Teruaki, K., Homma, K. and Yokoyama, A. (2010) Ciliated muconodular papillary tumor of the lung; A newly defined low-grade malignant tumor. Interactive Cardiovascular and Thoracic Surgery, 11, 685-687. doi:10.1510/icvts.2009.229989

[3] Hendrickson, M.R. and Kempson, R.L. (1983) Ciliated carcinoma; A variant of endometrial adenocarcinoma: A report of 10 cases. International Journal of Gynecological Pathology, 2, 1-12. doi:10.1097/00004347-198301000-00001

[4] Eichhorn, J.H. and Scully, R.E. (1996) Endometriod ciliated-cell tumors of the ovary: A report of five cases. International Journal of Gynecological Pathology, 3, 24856. doi:10.1097/00004347-199607000-00010

[5] Gupta, P.K., Albritton, N., Erozan, Y.S. and Frost, J.K. 
(1985) Occurrence of cilia in exfoliated ovarian adenocarcinoma cells. Diagnostic Cytopathology, 3, 228-231. doi:10.1002/dc.2840010311

[6] Nakamura, S., Koshikawa, T., Sato, T., Hayashi, K. and Suchi, T. (1992) Extremely well differentiated papillary adenocarcinoma of the lung with prominent cilia formation. Acta Pathologica Japonica, 10, 745-750.

[7] Imai, T., Suga, M., Kaimori, M., Hiyama, M., Yokoyama, K. and Kurotaki, H. (2010) Peripheral pulmonary papillary adenocarcinoma with prominent cilia. Acta Cytologica, 5, 949-957.
[8] Ross, H.M., Daniel, H.D.J., Vivekanandan, P., Kan- nahgai, R., Matthew, M.Y., Wu, T-T., Makhlouf, H.R. and Torbenson, M. (2011) Fibrolamellar carcinomas are positive for CD68. Modern Pathology, 24, 390-395. doi:10.1038/modpathol.2010.207

[9] Takashi, N., Kodama, T., Tsumuraya, M., Shimosato, Y. and Kameya, T. (1985) S-100 protein-positive Langerhans cells in various human lung cancers, especially in peripheral adenocarcinomas. Virchows Archiv A, 7, 177189. doi:10.1007/BF00737075 